\title{
Role for phosphatidylinositol 4-kinase III $\beta$ in cardiac metabolic diseases
}

Citation for published version (APA):

Sun, A. (2020). Role for phosphatidylinositol 4-kinase III in cardiac metabolic diseases. [Doctoral Thesis, Maastricht University]. Gildeprint Drukkerijen. https://doi.org/10.26481/dis.20200908as

Document status and date:

Published: 01/01/2020

DOI:

10.26481/dis.20200908as

Document Version:

Publisher's PDF, also known as Version of record

\section{Please check the document version of this publication:}

- A submitted manuscript is the version of the article upon submission and before peer-review. There can be important differences between the submitted version and the official published version of record.

People interested in the research are advised to contact the author for the final version of the publication, or visit the DOI to the publisher's website.

- The final author version and the galley proof are versions of the publication after peer review.

- The final published version features the final layout of the paper including the volume, issue and page numbers.

Link to publication

\footnotetext{
General rights rights.

- You may freely distribute the URL identifying the publication in the public portal. please follow below link for the End User Agreement:

www.umlib.nl/taverne-license

Take down policy

If you believe that this document breaches copyright please contact us at:

repository@maastrichtuniversity.nl

providing details and we will investigate your claim.
}

Copyright and moral rights for the publications made accessible in the public portal are retained by the authors and/or other copyright owners and it is a condition of accessing publications that users recognise and abide by the legal requirements associated with these

- Users may download and print one copy of any publication from the public portal for the purpose of private study or research.

- You may not further distribute the material or use it for any profit-making activity or commercial gain

If the publication is distributed under the terms of Article $25 \mathrm{fa}$ of the Dutch Copyright Act, indicated by the "Taverne" license above, 


\section{Summary}

Glucose and long chain fatty acids (LCFA) are the major energy substrates of the heart. Their uptake into cardiac myocytes is regulated by the membrane transporters GLUT4 and CD36, respectively. In the healthy heart, there is a balance between glucose and LCFA utilization, which is crucial for the heart to work properly. Departure from this balance is associated with cardiac diseases. Therefore, rebalancing substrate utilization could be a suitable strategy to prevent and/or regress cardiac diseases.

In the development of diabetic cardiomyopathy, CD36 permanently relocates to the sarcolemma, which increases LCFA uptake and, subsequently, myocellular lipid accumulation, eventually leading to inhibition of insulin signaling. As a result, insulin-stimulated myocellular GLUT4 translocation and glucose uptake are impaired, i.e., insulin resistance. Finally, the lipidinduced insulin resistance leads to heart dysfunction. Because insulin-stimulated GLUT4 translocation is defective in the type 2-diabetic heart, signaling pathways involved in contraction-induced GLUT4 translocation may contain suitable targets to increase cardiac glucose utilization during insulin resistance. One such kinase is protein kinase D1 (PKD1), a key player of contraction-induced GLUT4 translocation but not involved in contraction-induced CD36 translocation. However, PKD1 is also a major player in the development of cardiac hypertrophy, suggesting that, taken together, targeting PKD1 may not be beneficial to treat the diabetic heart.

Chapter 2 reviews the acute and chronic activation and regulatory functions of PKD signaling in both the healthy and hypertrophic heart, and the possibility of targeting this kinase as a novel strategy against cardiac diseases.

Given that PKD1 is also involved in cardiac hypertrophy, signaling modules downstream of PKD1 involved in contraction-induced GLUT4 translocation, but not triggering the undesired hypertrophic actions of PKD1 would be a preferred target to increase cardiac glucose utilization during insulin resistance, thus against diabetic cardiomyopathy. For this, phosphatidylinositol4-kinase-III $\beta$ (PI4KIII $\beta$ ), a lipid kinase downstream of PKD1, could be an alternative.

To study the role of PI4KIII $\beta$ in contraction-induced glucose uptake in cardiomyocytes, we aimed not only to genetically silence or pharmacologically inhibit PI4KIII $\beta$, but also to overexpress PI4KIII $\beta$ wild type, phosphorylation site mutant Ser294Ala (S294A) and kinasedeficient mutant Asp656Ala (D656A) in isolated adult rat cardiomyocytes. To enable this approach, in Chapter 3, we generated adenovirus encoding full-length human PI4KIII $\beta$ (wild type, S294A, D656A). 
Appendices

In Chapter 4, we evaluated whether PI4KIII $\beta$ is involved in myocellular GLUT4 translocation as induced by contraction or oligomycin ( an $\mathrm{F}_{1} \mathrm{~F}_{0}$-ATP synthase inhibitor that is activating contraction signaling), and whether it is a suitable anti-diabetic target. Pharmacological targeting, with compound MI14, or genetic silencing of PI4KIII $\beta$ inhibited contraction/oligomycin-stimulated GLUT4 translocation and glucose uptake in cardiomyocytes, but did not affect CD36 translocation nor fatty acid uptake. Addition of the PI4KIII $\beta$ enzymatic reaction product phosphatidylinositol-4-phosphate restored oligomycin-stimulated glucose uptake in the presence of MI14. PKD1-induced PI4KIII $\beta$ activation involves Ser294 phosphorylation and altered its localization with unchanged enzymatic activity. Adenoviral PI4KIII $\beta$ overexpression stimulated basal glucose uptake. Importantly, PI4KIII $\beta$ overexpression did not activate hypertrophic signaling, indicating that, unlike PKD1, PI4KIII $\beta$ is selectively involved in GLUT4 translocation. Finally, PI4KIII $\beta$ overexpression prevented insulin resistance in lipid-overexposed cardiomyocytes. Collectively, we identify PI4KIII $\beta$ as positive regulator of GLUT4 translocation and as potential target against diabetic cardiomyopathy.

Chronic pressure overload leads to cardiac hypertrophy which results in contractile dysfunction and eventually heart failure. In the development of cardiac hypertrophy, the shift in substrate preference towards glucose uptake has been considered as the main contributor. Therefore, inhibition of signaling kinases that regulate glucose uptake might be a promising strategy to rescue cardiac hypertrophy and subsequent contractile dysfunction. PKD1, which regulates contraction-induced glucose uptake, has been studied as a potential target to prevent cardiac hypertrophy (Chapter 2). However, PKD1 is also directly involved in cardiac hypertrophy via histone deacetylase-5 (HDAC5), making it difficult to distinguish whether the beneficial action of PKD1 inhibition is caused by inhibition of its hypertrophic signaling pathway or due to inhibition of glucose uptake. In Chapter 4, we identified PI4KIII $\beta$, a downstream kinase of PKD1, as a key positive regulator in contraction-induced glucose uptake but not a player in cardiac hypertrophy. In Chapter 5, we aimed to determine whether inhibition of PI4KIII $\beta$ would downregulate enhanced glucose uptake in the pressure-overloaded hypertrophic heart and thus prevent worsening of cardiac hypertrophy and the concomitant contractile dysfunction. We used $\alpha 1$-adrenoceptor agonist phenylephrine (PE) as a pressure overload-induced hypertrophy-mimetic stimulus, leading to increased expression of the hypertrophic marker brain natriuretic peptide (BNP) and increased phosphorylation of signaling kinases related to hypertrophy (including mammalian target of rapamycin complex1 (mTORC1), extracellular signal-regulated kinase 1/2 (ERK 1/2), PKD1, and HDAC5). Moreover, chronic PE stimulation caused enhanced protein synthesis and glucose uptake, and impaired contractile 
function. Co-incubation of specific PI4KIII $\beta$ inhibitor, MI14, inhibited PE-stimulated glucose uptake and decreased PE-stimulated phosphorylation of PKD1 at ser916 and HDAC5 at ser498, but did not affect PE-induced BNP expression nor protein synthesis. Moreover, MI14 restored PE-induced contractile dysfunction. Taken together, these findings suggest that although decreasing glucose uptake cannot prevent all the aspects of cardiac hypertrophy, MI14-mediated PI4KIII $\beta$ inhibition is a promising strategy to preserve contractile function in the pressureoverloaded hypertrophic heart.

Altogether, this thesis has revealed a key role for PI4KIII $\beta$ in myocellular contraction-induced GLUT4 translocation and as therapeutic target for cardiac metabolic diseases. These findings may yield novel strategies by which rebalancing myocardial substrate utilization via PI4KIII $\beta$ could help prevent and/or regress both diabetic cardiomyopathy and cardiac hypertrophyinduced contractile dysfunction. 
Appendices

\section{Samenvatting}

Glucose en lang-keten vetzuren zijn de belangrijkste energierijke substraten voor het hart. De opname van deze substraten door hartspiercellen wordt gereguleerd door respectievelijk de membraan-transporters GLUT4 en CD36. In het gezonde hart is er een zekere balans tussen het gebruik van glucose en dat van vetzuren, die cruciaal is voor een goede werking van het hart. Afwijkingen van deze balans zijn geassocieerd met hartaandoeningen. Op grond daarvan is betoogd dat het opnieuw in evenwicht brengen van het substraatgebruik een geschikte strategie kan zijn om hartaandoeningen te voorkomen en wellicht ook te behandelen.

In het gezonde hart is de vetzuurtransporter CD36 deels aanwezig in een celcompartiment (endosomen) van waaruit het naar de celmembraan (sarcolemma) kan migreren om dan de vetzuuropname te stimuleren. Komt het hart in rust, dan vindt het omgekeerde proces plaats (internalisatie van CD36). Bij de ontwikkeling van diabetische cardiomyopathie zien we echter dat CD36 permanent verhuist naar de sarcolemma; dit zorgt voor een ongecontroleerde verhoging van de vetzuuropname. Vervolgens leidt deze CD36 re-locatie tot accumulatie van lipiden in het hart en uiteindelijk tot remming van de insuline-signalering. Dientengevolge zijn de insuline-gestimuleerde GLUT4-translocatie en glucose-opname in hartspiercellen verminderd; deze conditie wordt aangeduid als insuline-resistentie. Tenslotte leidt een dergelijke lipide-geïnduceerde insuline-resistentie tot vermindering van de contractie van de hartspiercellen, en dus een verminderde pompfunctie van het hart.

Beperking van de vetzuuropname òf verhoging van de (GLUT4-gemedieerde) glucose opname zou de substraatbalans en daarmee de pompfunctie van het diabete hart kunnen herstellen. Aangezien in het type-2 diabete hart de insuline-gestimuleerde GLUT4-translocatie defect is, zullen signaalroutes betrokken bij contractie-gestimuleerde GLUT4-translocatie geschiktere aangrijpingspunten zijn om het verbruik van glucose door het hart tijdens insulineresistentie te verhogen. Eén zo'n kinase is proteïne kinase D1 (PKD1), een belangrijke speler in contractie-gestimuleerde GLUT4-translocatie maar niet betrokken bij contractiegestimuleerde CD36-translocatie. PKD1 is echter ook betrokken bij de ontwikkeling van abnormale hartvergroting (hypertrofie), waardoor manipulaties ter activatie van PKD1 mogelijk niet gunstig zijn voor de behandeling van het diabetische hart.

Hoofdstuk 2 is een review die de acute en chronische activering en regulerende functies van PKD-signalering in zowel het gezonde als hypertrofische hart bespreekt. Verder evalueert deze review de mogelijkheid om activatie van deze of van gerelateerde kinases als een nieuwe strategie tegen hartaandoeningen te gebruiken. 
Aangezien PKD1 ook betrokken is bij hypertrofie van het hart, zouden signaalmodules stroomafwaarts van PKD1, die betrokken zijn bij contractie-geïnduceerde GLUT4-translocatie, maar niet bij de ongewenste hypertrofische invloeden van PKD1, een veel geschikter aangrijpingspunt kunnen zijn om het glucose-verbruik in het hart te verhogen tijdens insulineresistentie. Hierdoor zou diabetische cardiomyopathie tegengegaan kunnen worden. Eén van deze mogelijke aangrijpingspunten is fosfatidylinositol-4-kinase-III $\beta$ (PI4KIII $\beta$ ), een lipidekinase direct stroomafwaarts van PKD1.

Om de rol van PI4KIII $\beta$ bij contractie-gestimuleerde glucoseopname in hartspiercellen te bestuderen, wilden we PI4KIII $\beta$ zowel genetisch kunnen uitschakelen en farmacologisch remmen. Tevens wilden we het wildtype PI4KIII $\beta$, alsook de fosforylatie-deficiënte mutant Ser294Ala (S294A) en een kinase-deficiënte mutant Asp656Ala (D656Ala) tot overexpressie kunnen brengen in geïsoleerde volwassen hartspiercellen van de rat. Om deze aanpak mogelijk te maken, hebben we in Hoofdstuk 3 adenovirus-constructen gegenereerd van het volledige menselijke PI4KIII $\beta$ gen en van de twee mutanten (wildtype, S294A, D656A).

In Hoofdstuk 4 hebben we geëvalueerd of PI4KIII $\beta$ betrokken is bij contractie-gestimuleerde GLUT4-translocatie in het hart en bij GLUT4 translocatie veroorzaakt door oligomycine (een $\mathrm{F}_{1} \mathrm{~F}_{0}$-ATP-synthase-remmer die contractiesignalering activeert). Ook hebben we onderzocht of PI4KIII $\beta$ een geschikt anti-diabetisch aangrijpingspunt is. Zowel farmacologische remming met de moleculaire verbinding MI14 alsook genetische uitschakeling van PI4KIII $\beta$ remde de contractie/oligomycine-gestimuleerde GLUT4 translocatie en glucoseopname in hartspiercellen, maar had geen invloed op CD36 translocatie en vetzuuropname. Toevoeging van PI4KIII $\beta$ 's enzymatische reactie-product fosfatidylinositol-4-fosfaat herstelde de oligomycine gestimuleerde glucose-opname in aanwezigheid van MI14. Het mechanisme van contractie/oligomycine-gestimuleerde PI4KIII $\beta$-activering omvat Ser294-fosforylering en een veranderde lokalisatie van dit eiwit met een onveranderde enzymatische activiteit. Overexpressie van adenoviraal PI4KIII $\beta$ stimuleerde de basale glucose-opname. Belangrijk is dat overexpressie van PI4KIII $\beta$ niet de hypertrofische signalering in het hart activeerde; dit betekent dat, in tegenstelling tot PKD1, PI4KIII $\beta$ selectief betrokken is bij GLUT4-translocatie. Tenslotte voorkwam PI4KIII $\beta$-overexpressie insuline-resistentie in overmatig aan lipiden blootgestelde hartspiercellen. Samengevat kunnen we hieruit concluderen dat PI4KIII $\beta$ een positieve regulator is van GLUT4 translocatie en dus toegepast kan worden als potentieel aangrijpingspunt tegen diabetische cardiomyopathie.

Chronische overbelasting leidt tot hypertrofie van het hart, wat leidt tot contractiele disfunctie en uiteindelijk tot hartfalen. Bij de ontwikkeling van hypertrofie van het hart wordt de 
Appendices

verschuiving in substraatvoorkeur naar glucose-opname beschouwd als de belangrijkste oorzaak. Daarom kan remming van signalerings-kinases betrokken bij glucose-opname een veelbelovende strategie zijn om hypertrofie van het hart en de daaropvolgende contractiele disfunctie tegen te gaan. In dit verband is PKD1, een positieve regulator van de contractiegestimuleerde glucose-opname, onderzocht als een potentieel aangrijpingspunt om cardiale hypertrofie te voorkomen (hoofdstuk 2). Echter, PKD1 is ook direct betrokken bij het ontstaan van hypertrofie van het hart, namelijk via het enzym histon-deacetylase-5 (HDAC5). Hierdoor is het moeilijk onderscheid te maken of de gunstige werking van PKD1-remming wordt veroorzaakt door remming van de hypertrofische signaalroute òf door remming van de glucoseopname. In Hoofdstuk 4 konden we aantonen dat PI4KIII $\beta$, stroomafwaarts gelegen van PKD1, een belangrijke positieve regulator is van contractie-gestimuleerde glucoseopname, maar niet is betrokken bij het ontstaan van hart-hypertrofie. In Hoofdstuk $\mathbf{5}$ hebben we getracht te bepalen of remming van PI4KIII $\beta$ de verhoogde glucose-opname in het overbelaste hypertrofische hart zou verlagen en zodoende de verergering van hart-hypertrofie en de daarmee gepaard gaande contractiele disfunctie zou voorkomen. We gebruikten een $\alpha 1$-adrenoceptoragonist phenylephrine (PE) als een hypertrofie-mimetische stimulus in vitro in gekweekte hartspiercellen. Dit leidde tot verhoogde expressie van het hypertrofische marker-eiwit breinnatriuretisch peptide (BNP) en verhoogde fosforylering van signalerings-kinases gerelateerd aan hypertrofie (inclusief mammalian-target-of-rapamycin-complex-1; mTORC1, extracellulair signaal-gereguleerd kinase-1/2; ERK-1/2, PKD1) en als laatste tot fosforylering van het hypertrofie-repressor eiwit HDAC5. Bovendien leidde chronische PE-stimulatie tot een toegenomen eiwitsynthese en glucoseopname en een verminderde contractiele functie. Coincubatie met de specifieke PI4KIII $\beta$-remmer, MI14, remde de PE-gestimuleerde glucoseopname en verminderde de PE-gestimuleerde fosforylering van PKD1-Ser916 en HDAC5-ser498, maar had geen invloed op de PE-gestimuleerde BNP-expressie en eiwitsynthese. Bovendien herstelde MI14 de PE-gestimuleerde contractiele disfunctie. Samengevat suggereren deze bevindingen dat, hoewel een remming van de glucose-opname niet alle aspecten van hart-hypertrofie kan voorkomen, PI4KIII $\beta$-remming middels MI14 een veelbelovende strategie is om de contractiele functie in het overbelaste hypertrofische hart te behouden.

In algemene termen heeft dit proefschrift een sleutelrol onthuld voor PI4KIII $\beta$ in contractiegestimuleerde GLUT4 translocatie en lijkt PI4KIII $\beta$ zeer geschikt als therapeutisch aangrijpingspunt voor metabole ziekten van het hart. Op grond van deze bevindingen kunnen nieuwe strategieën worden ontworpen waarmee het opnieuw in evenwicht brengen van de 
substraat-balans van het hart via PI4KIII $\beta$-manipulatie zowel diabetische cardiomyopathie alsmede hypertrofie-geïnduceerde contractiele disfunctie kan helpen voorkomen en/of genezen. 


\section{总结}

葡萄糖和脂肪酸是心脏的主要供能底物。心肌细胞的葡萄糖和脂肪酸摄取分别由细 胞膜上的转运体 GLUT4 和 CD36 介导调节。在健康的心脏中, 葡萄糖和脂肪酸的利用 存在着一个平衡, 对心脏的正常运转至关重要。这种平衡的失调与心脏病有着密切的联 系。因此，重新平衡底物的利用可以作为预防和/或治疗心脏病的一个可行的策略。

在糖尿病型心肌病的形成过程中，CD36 永久地迁移定位到肌膜上，增加了脂肪酸 的摄取, 接着增加了细胞内脂类的存储, 最后导致了胰岛素信号通路的抑制: 由胰岛素 激发的肌细胞 GLUT4 转运和葡萄糖摄取被破坏, 引起胰岛素抵抗。最终, 脂类引发的 胰岛素抵抗造成了心功能障碍。在二型糖尿病的心脏中, 由胰岛素激发的 GLUT4 转运 存在障碍, 因此收缩引发的 GLUT4 转运中的信号通路可能含有合适的靶点, 来增加在 胰岛素抵抗的情况下的心脏葡萄糖利用。其中, 参与收缩引发的 GLUT4 转运但不参与 收缩引发的 CD36 转运的蛋白激酶 D1（PKD1）是这样的一个靶点。然而, PKD1 也是 心脏肥大形成的一个重要参与者, 因此, 中上所述, PKD1 可能不是一个治疗糖尿病型 心肌病的有益靶点。

第二章评述了在健康和肥大心脏中 PKD 的急性和慢性激活和调节作用，以及将其 作为治疗心脏病的靶点的可能性。

考虑到 PKD1 也参与了心脏肥大，因此参与了收缩引发的 GLUT4 转运而不引起 PKD1 所引起的心脏肥大的 PKD1 的下游通路可能是增加在胰岛素抵抗情况下的心脏葡 萄糖利用的更好的靶点。其中，PKD1 下游的脂质激酶：磷脂酰肌醇-4-激酶-III $\beta$ （PI4KIII $\beta ）$ 可以作为一个候选。

为了研究心肌细胞中 PI4KIII $\beta$ 在收缩引发的葡萄糖摄取中的作用, 我们不止要在基 因层面沉默或药理上抑制 PI4KIII $\beta$, 还需要在分离的成年大鼠原代心肌细胞中过表达野 生型, 磷酸化位点突变型 (S294A) 和激酶缺陷型（D656A）的 PI4KIII $\beta$ 。因此, 在第 三章中, 我们构建获得了编码人类全长的 PI4KIII $\beta$ （包括野生型, S294A 和 D656A）的 腺病毒。

在第四章中, 我们检测了 PI4KIII $\beta$ 是否参与了肌细胞中收缩或寡霉素 (oligomycin, $\mathrm{F}_{1} \mathrm{~F}_{0}$ 型 $\mathrm{ATP}$ 合成酶抑制剂, 可以激活收缩通路) 引发的 GLUT4 转运, 是否是一个合适 的抗糖尿病靶点。通过化合物 MI14 在药理上抑制 PI4KIII $\beta$ 和基因沉默 PI4KIII $\beta$, 均抑 制了心肌细胞中收缩或寡霉素激发的 GLUT4 转运和葡萄糖摄取, 但对 CD36 转运和脂 肪酸摄取没有作用。添加 PI4KIII $\beta$ 的产物：磷脂酰肌醇-4-磷酸，恢复了 MI14 所抑制的 寡霉素激发的葡萄糖摄取。PKD1 引发的 PI4KIII $\beta$ 激活涉及到第 294 位的丝氨酸的磷酸 化和细胞定位的改变, 但不改变酶活。通过腺病毒过表达 PI4KIII $\beta$ 增加了基础水平的葡 萄糖摄取。重要的是, PI4KIII $\beta$ 的过表达没有激活肥大通路, 因此, 与 PKD1 不同, PI4KIII $\beta$ 是有选择性地参与了 GLUT4 转运。最后, PI4KIII $\beta$ 的过表达避免了过度暴露在 脂质条件下的心肌细胞的胰岛素抵抗的形成。综上, 我们鉴定了 PI4KIII $\beta$ 参与了 GLUT4 转运的正向调节并可以作为抗糖尿病型心肌病的潜在靶点。

慢性压力过载导致心脏肥大，造成收缩功能障碍和最终的心衰。底物偏好向葡萄糖 摄取转变被认为是心脏肥大形成的主要原因。因此，抑制参与葡萄糖摄取的信号激酶可 能是一个有希望挽救心脏肥大及后续的收缩功能障碍的策略。参与收缩激发的葡萄糖摄 取的 PKD1 被研究认为可以作为预防心脏肥大的潜在靶点（第二章）。然而, PKD1 还 通过组蛋白脱乙酰基酶 5（HDAC5）直接参与了心脏肥大的形成，因此很难去分辨通过 抑制 PKD1 而得到的抗心脏肥大的作用是由于抑制了肥大信号通路还是因为抑制了葡萄 糖摄取。在第四章中, 我们鉴定了 PI4KIII $\beta$, 作为 PKD1 的下游激酶, 在收缩引发的葡 萄糖摄取中是一个重要的正向调节因子, 而不参与心脏肥大。在第五章中，我们意在确 
定是否抑制 PI $4 \mathrm{KIII} \beta$ 可以下调压力过载造成的肥大心脏中升高的葡萄糖摄取, 并因此避 免心脏肥大的恶化及伴随的收缩功能障碍。我们利用 $\alpha 1$-肾上腺素受体激动剂: 苯肾上 腺素（phenylephrine, PE）作为压力过载造成的肥大的模拟刺激, 引起肥大标志物脑钠 肽 (BNP) mRNA 水平的升高, 及肥大相关信号激酶（包括雷帕霉素复合物的哺乳动物 靶点 1（mTORC1），细胞外信号调节激酶 $1 / 2(E R K 1 / 2)$, PKD1 和 HDAC5）的磷酸 化增强。此外, PE 的慢性刺激增加了蛋白质合成和葡萄糖摄取, 并破坏了收缩功能。

与 PI 4 KIII $\beta$ 的特定抑制剂 MI14 共睬育抑制了 PE 激发的葡萄糖摄取, 降低了 PE 激发的 PKD1 第 916 位丝氨酸和 HDAC5 第 498 位丝氨酸的磷酸化, 但是对 PE 激发的 BNP 表 达和蛋白质合成没有影响。除此之外, MI14 修复了 PE 引起的收缩功能障碍。综上, 这 些发现表明, 虽然降低葡萄糖摄取不能完全避免心脏肥大的形成, 但是 MI14 介导的 PI4KIII $\beta$ 抑制作用是一个有希望保持压力过载造成的肥大心脏的收缩功能的策略。

总的来说, 本论文揭示了 PI4KIII 在肌细胞收缩引发的 GLUT4 转运中的关键作用 并可以作为抗心脏代谢疾病的靶点。这些发现提供了一个新的策略, 即通过 PI IKIII $\beta$ 重 新平衡心肌底物的利用, 可以帮助预防和/或治疗糖尿病型心肌病和心脏肥大造成的收 缩功能障碍。 